\title{
Optimal Allocation of the Distributed Generations using AMPSO Wang Ya-fang ${ }^{1, a}$, Wang Tao $^{2, b}$ and Ren Xiao ${ }^{3, c}$ \\ ${ }^{1,3}$ College of Electrical Engineering, Guizhou University, Guiyang 550003, China \\ ${ }^{2}$ Anren power supply bureau of Hunan SGCC, Anren 423600, China \\ aneneqiu@163.com, b361725319@qq.com, 275323875@qq.com
}

Keywords: DG, optimal allocation, AMPSO, fuzzy set theory

\begin{abstract}
This paper presented an application of Adaptive Mutation Particle Swarm Optimization (AMPSO) in optimal allocation of Distributed Generation (DG) in distribution power networks. The multiple-objective function to be optimized included two objectives: real power loss and total voltage deviation. Through fuzzy set theory, the multiple-objective optimization problem had been transformed to single objective comprehensive optimization with membership degree. This method was tested on standard IEEE 33 bus using MATLAB 2010. The results showed that the proposed method was accurate and fast.
\end{abstract}

\section{Introduction}

The characteristics of DG have high efficiency, flexible generating kinds, environmental protection, small investment and so on. Optimal allocation of DG research contributed to the safe and stable operation of the distribution network, and saved investment, and reduced the energy consumption of electric power, and improved power supply reliability and flexibility.

Aiming at the optimal allocation of DG, scholars at home and abroad had carried out a series of research which put forward different optimal allocation models of the DG. References [1] and [2] proposed to consider environmental factors of DG configuration model. Reference [3] established three comprehensive cost of objective function which were the distributed generation construction cost and network operation cost, improving voltage quality efficiency and environmental benefits reducing the carbon emissions. Reference [4] proposed a " $2 / 3$ rule" to determine the optimal location of DG in distribution network, the rule was simple, and easy to use, but the rule was only applicable to uniformly distributed load along the feeder which was a unified load characteristic of radial feeder.

\section{Optimal Formulation}

Objective Functions. With proper planning, the integration of distributed generations in a distribution system would lead to enhancement in the network performance in terms of voltage profile improvement and reduction in line losses.

The first objective distribution system real power loss ${ }^{[5]}$ is given by,

$$
\min P_{N}=\sum_{i=1}^{N}\left|I_{i}\right|^{2} R_{i}
$$

The second objective is to minimize the total voltage deviation, it can help to guarantee the better voltage level in distribution power system. The total voltage deviation ${ }^{[6]}$ is given by,

$$
\operatorname{Det} V=\sum_{i=1}^{n}\left|U_{b}-U_{i}\right|
$$

Constraints. Constraints including inequality constraints and equality constraints, equality constraint is the power balance equation, inequality constraints are given by,

$U_{i}^{\min } \leq U_{i} \leq U_{i}^{\max }$

$I_{i} \leq I_{i}^{\max }$

$$
\sum S_{D G} \leq 0.4 \sum S_{L D}
$$

Multiple-objective Optimization by using Fuzzy Set Theory. Fuzzy set theory is suitable for 
describe uncertainty and dealing with different dimensions of multiple-objective optimization problem, the most important thing is to build the objective function of the membership function, after processing the objective function valued in the real unit interval $[0,1]$. According to the established objective function using piecewise linear function as membership function of each objective. Membership function is given by,

$$
\mu_{i}=\left\{\begin{array}{l}
1, f_{i} \leq f_{i}^{s} \\
1-\frac{f_{i}-f_{i}^{s}}{f_{i}^{m}-f_{i}^{s}}, f_{i}^{s}<f<f_{i}^{m} \\
0, f_{i} \geq f_{i}^{m}
\end{array}\right.
$$

The satisfaction degree for the above two objective function is given by,

$$
S=\min \left\{\mu_{1}, \mu_{2}\right\}
$$

\section{Optimal Algorithm}

Adaptive Mutation Particle Swarm Optimization(AMPSO). In particle PSO, a solution of each particle represents the specific optimization problems, there is a velocity vector $\mathrm{v}_{\mathrm{i}}$ and the position vector $\mathrm{x}_{\mathrm{i}}$, by comparing determined by the fitness function to adapt to the value of the particles, in the process of iterative optimization, particle by tracking individual optimal solution $\mathrm{P}_{\mathrm{i}}$ and the global optimal solution $\mathrm{P}_{\mathrm{g}}$, and constantly update their location and velocity, until we find the optimal solution, the position and velocity updating formula is as follows:

$$
\left\{\begin{array}{l}
v_{i}^{k+1}=\omega v_{i}^{k}+c_{1} r_{1}\left[P_{i}^{k}-x_{i}^{k}\right]+c_{2} r_{2}\left[P_{g}^{k}-x_{i}^{k}\right] \\
x_{i}^{k+1}=x_{i}^{k}+v_{i}^{k+1}
\end{array}\right.
$$

The $\mathrm{k}^{\text {th }}$ iteration of inertia weight value for $\omega$ is as follows:

$$
\omega_{k}=\omega_{\min }+\frac{\omega_{\max }-\omega_{\min }}{N}
$$

AMPSO using swarm variance to judge the degree of convergence of the algorithm, thus the adaptive change of mutation probability, and adopt the method of random disturbance to mutation of global extreme value, can effectively avoid the premature problem.

The fitness variance $\sigma^{2}$ of swarm in $\mathrm{k}^{\text {th }}$ iteration is given by,

$$
\sigma^{2}=\sum_{i=1}^{n}\left(\frac{f_{i}-f_{a v g}}{f}\right)^{2}
$$

The value of $\sigma^{2}$ smaller, the algorithm convergence, and vice is in the stage of random search algorithm. Mutation probability should adaptive change with $\sigma^{2}$, the $\mathrm{k}^{\text {th }}$ iteration of mutation probability $^{[7]}$ is as follows:

$$
P_{k}=\left(P_{\max }-P_{\min }\right)\left(\sigma^{2} / n\right)^{2}+\left(P_{\min }-P_{\max }\right)\left(2 \sigma^{2} / n\right)+P_{\max }
$$

Adopt the method of random disturbance on $P_{g}$ 's mutation operation, generate a random number $r \in[0,1]$, and when $r<P_{k}$ to Gauss mutation:

$$
P_{g}=P_{g}(1+0.5 \eta)
$$

Optimal Allocation of DG Based on AMPSO. The methodology based on multiple-objective AMPSO for optimal allocation of DG in power system is as follows:

Step1: Initialization. Input load data and line data of distribution network, initialize the PSO parameters.

Step2: Initialize the particle swarm. Within the prescribed scope of particle position and velocity, randomly generated particle's position and velocity of the initial value.

Step3: Power flow calculation. Calculate each particle fitness.

Step4: Update the individual optimal solution and the global optimal solution.

Step5: Calculate the current group fitness variance and mutation probability.

Step6: Generate a random number $r \in[0,1]$, and when $r<P_{k}$ to mutation, otherwise into step 7. 
Step7: Adjust the particle's position and velocity

Step8: Determine whether meet the termination conditions. If meet the conditions then output, otherwise into step 7.

Step9: Output the global optimal solution.

\section{Results and Discussion}

The proposed method has been tested on IEEE 33-bus radial distribution system(Fig. 1) having following characteristics:Number of buses $=33$; Number of lines $=32$; Base Voltage $=12.66 \mathrm{KV}$; Total Active Load=3715.0kW; Total Reactive Load=2300.0kvar. In AMPSO, Swarm Size=50; Particle Dimension=32; Largest Number of Iterations $=300 ; c_{1}=c_{2}=2 ; \omega_{\min }=0.4 ; \omega_{\max }=0.9$.

The test system is simulated in MATLAB 2010 and the test results are as shown below.

The Optimal results and the location of optimal satisfaction are shown in Tab.1 and Fig.2. The bus voltage comparison before optimization and after the optimization in IEEE 33-bus system is shown in Fig.3. As shown in Fig.3, the voltage magnitude has been improved since the DG units provide active power to the network. Through Tab.1 also can find that real power loss and total voltage deviation have been improved a lot with DG.

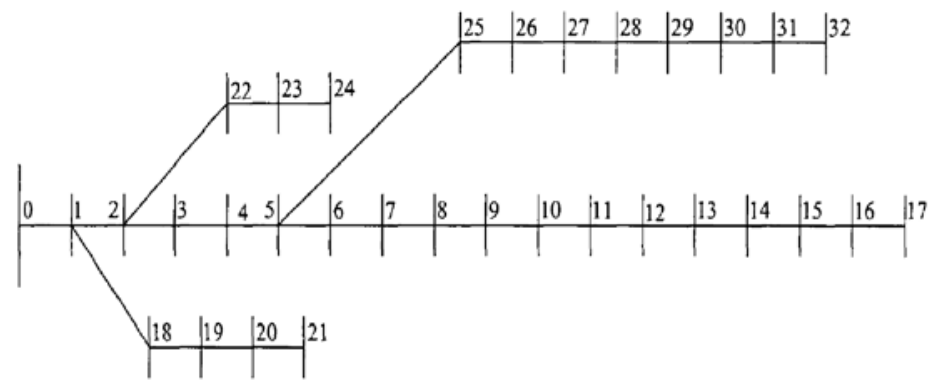

Fig. 1 IEEE 33-bus system

Tab. 1 Optimal results

\begin{tabular}{|c|c|c|c|}
\hline Serial Number & $\begin{array}{c}\text { DG Location } \\
\text { and DG Size }(\mathrm{kW})\end{array}$ & $\begin{array}{c}\text { Real Power Loss } \\
(\mathrm{kW})\end{array}$ & $\begin{array}{c}\text { Total Voltage } \\
\text { Deviation }\end{array}$ \\
\hline 1 & - & 180.909 & 0.02929 \\
\hline 2 & $\begin{array}{c}\text { Bus 15 with 600 } \\
\text { Bus 27 with 600 }\end{array}$ & 55.096 & 0.01128 \\
\hline \multicolumn{2}{|c|}{$\%$ Improvement } & 69.54 & 61.49 \\
\hline
\end{tabular}

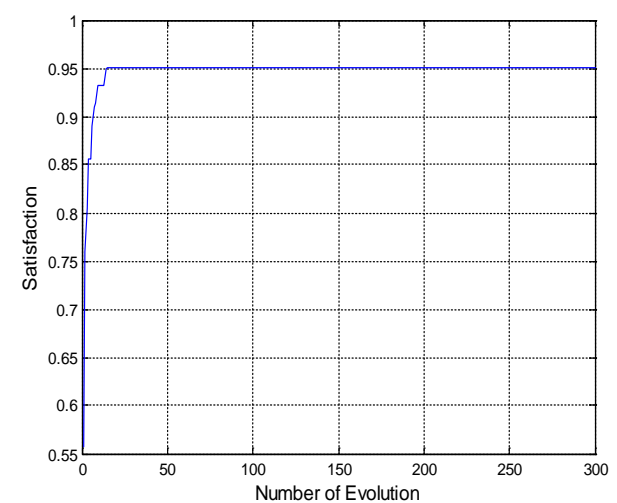

Fig. 2 Location of Optimal Satisfaction

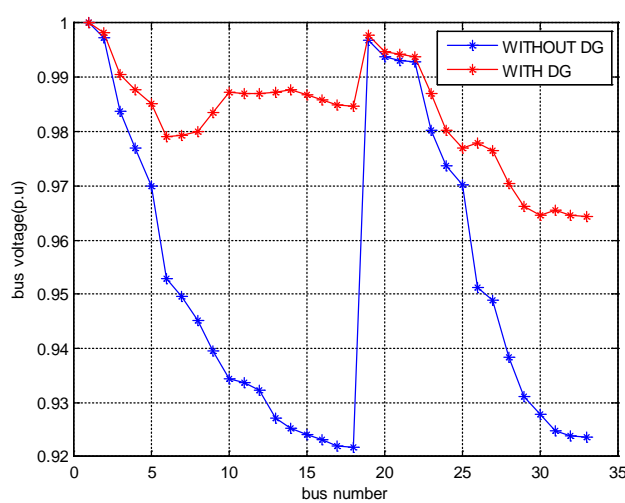

Fig. 3 Comparison of bus voltage without/with DG

\section{Conclusion}

The proposed method based on AMPSO for optimal allocation of DG considering multiple-objective optimization function which is combination of real power loss and total voltage deviation. It is apparent from the results that there is considerable reduction in real power loss and 
total voltage deviation. The results show that the method was accurate and fast.

\section{References}

[1] Zheng Zhang-hua, Ai Qian, GU Cheng-hong, et al. Multi-objective allocation of distributed generation considering environmental factor[J]. Proceedings of the CSEE, 2009, 29(13):23-28.

[2] Cui Hong, Guo Yi-yun, Xia Cheng-jun. Research on Optimal Allocation of Distributed Generation by Considering Environmental Benefits[J]. East China Electric Power, 2010, 38(12): 1968-1971.

[3] Wu Jia-sheng, AI Xin. Study on sitting and sizing of distribution generation with objective of low carbon[J]. Modern Electric Power, 2014, 31(1): 17-22.

[4] H.L.Willis, “Analytical methods and rules of thumb for modeling DG-distribution interaction,” in Proc. IEEE Power Eng. Soc. Summer Meeting, Jul. 2000, pp. 1643-1644.

[5] Wang Xing-wang, Qiu Xiao-yan. Distributed generation planning in distribution system based on modified particle swarm optimization algorithm[J]. Power System Protection and Control, 2009, 37(14): 16-20.

[6] GU Chen, LE Xiu-fan. Optimal allocation of distributed generation based on NSGA Modern electric power, 2014, 31(1): 17-22.

[7] Chen Gui-min, Qin Jian-yuan. Han Qi. Study on the Strategy of Decreasing Inertia Weight in Particle Swarm Optimization Algorithm[J]. Journal of Xi'an Jiaotong University, 2006, 40(1): 53-61. 